\title{
Is vancomycin MIC creep a worldwide phenomenon? Assessment of S. aureus vancomycin MIC in a tertiary university hospital
}

\author{
Silvestre Joana ${ }^{1,2^{*}}$, Póvoa Pedro ${ }^{1,2}$, Gonçalves Elsa ${ }^{3}$ and Martins Filomena ${ }^{3}$
}

\begin{abstract}
Background: Vancomycin is the primary treatment for infections caused by methicilin-resistant Staphylococcus aureus (MRSA). The association of vancomycin treatment failures with increased vancomycin minimum inhibitory concentration (MIC) is a well-recognized problem. A number of single-centre studies have identified progressive increases in glycopeptide MICs for S. aureus strains over recent years - a phenomenon known as vancomycin MIC creep. It is unknown if this is a worldwide phenomenon or if it is localized to specific centers.

Methods: The aim of this study was to evaluate the trend of vancomycin MIC for isolates of MRSA over a 3-year period in a tertiary university hospital in Portugal. MRSA isolates from samples of patients admitted from January 2007 to December 2009 were assessed. Etest method was used to determine the respective vancomycin MIC. Only one isolate per patient was included in the final analysis.

Results: A total of 93 MRSA isolates were studied. The vancomycin MICs were $0.75,1,1.5$ and $2 \mathrm{mg} / \mathrm{L}$ for 1 (1.1\%), 19 (20.4\%), 38 (40.9\%), 35 (37.6\%) isolates, respectively. During the 3 year period, we observed a significant fluctuation in the rate of MRSA with a vancomycin MIC > 1 mg/L (2007: 86.2\%; 2008: 93.3\%; 2009: 58.8\%, $p=0.002$ ). No MRSA isolate presented a MIC > $2 \mathrm{mg} / \mathrm{L}$.

Conclusions: We were unable to find in our institution data compatible to the presence of vancomycin MIC creep during the study period. This phenomenon seems not to be generalized; as a result each institution should systematically monitor MRSA vancomycin MIC over time.
\end{abstract}

\section{Background}

According to the Surveillance Network database, which collected laboratory data from 300 clinical microbiology laboratories across the United States, from 1998 to March 2005 with more than 3 million bacterial isolates, Staphylococcus aureus was the most prevalent (18.7\%) species isolated from inpatient specimens and the second most prevalent (14.7\%) species from outpatient specimens [1]. In 2005, MRSA rates were $59.2 \%, 55 \%$, and $47.9 \%$ for strains from non-intensive care unit (ICU) inpatients, ICU, and outpatients, respectively [1].

\footnotetext{
* Correspondence: joanapsilvestre@gmail.com

'Polyvalent Intensive Care Unit, São Francisco Xavier Hospital, CHLO, Lisbon, Portugal

${ }^{2}$ CEDOC, Faculty of Medical Sciences, New University of Lisbon, Lisbon, Portugal

Full list of author information is available at the end of the article
}

Although the proportion of Staphylococcus aureus bacteraemia due to MRSA is declining in many countries, data from the European Antimicrobial Resistance Surveillance System (EARSS) for 2010 showed that in more than one-third of countries the proportion remained $>25 \%$ [2]. The highest rates were reported from the Mediterranean countries, with Portugal being the only country showing MRSA rates above $50 \%[2,3]$. The Portuguese data were further confirmed by MeloCristino et al. in a recent study [4].

Vancomycin, a glycopeptide antimicrobial, was initially used to treat infections with penicillin-resistant Staphylococcus aureus before alternative, less-toxic drugs were introduced [5]. Subsequently this glycopeptide became vital for the treatment of infections with MRSA [5]. Vancomycin is now the $1^{\text {st }}$ line treatment for infections caused by MRSA [6]. However, vancomycin treatment

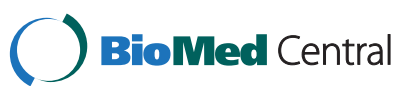


failure in MRSA is not uncommon, even when MRSA is susceptible to vancomycin.

There are a number of types of susceptibility test available. Traditionally, agar disk diffusion has been used to measure glycopeptide susceptibility, but now this method is not regarded as standard, since it does not measure the MIC. This method is not suitable for large antibiotic molecules, such as glycopeptides and daptomycin, because they diffuse too slowly into agar. An alternative method for measuring glycopeptide MIC is broth microdilution - the gold-standard test for measuring antibiotic MICs. Automated susceptibility testing systems are also widely used, but the performance of this methodology for measuring glycopeptide MICs has been frequently questioned. Etest has been developed as an accurate and easier agar plate method. An Etest strip, which contains a gradient of antibiotic, is placed on an inoculated agar plate and the pattern of bacterial growth is examined after 24 hours. Since the Etest uses a gradient of antibiotic concentration, it has greater precision than disc diffusion methods, allowing better ascertainment of the actual MIC.

There is a growing body of evidence showing a sustained increase in the MICs of glycopeptides against Staphylococcus aureus strains, a process referred to as "MIC creep", however with several conflicting results [7-9].

Thus, the primary objective of our study was to evaluate MIC trends for clinical MRSA isolates to vancomycin over a 3-year period in one hospital using the Etest.

\section{Methods}

This study was previously approved by the ethical committee of our institution; no informed consent was needed, according to the ethical principles of the declaration of Helsinki.

\section{Microorganisms}

Clinical MRSA isolates from cultures were collected from sequential individual patients at São Francisco Xavier Hospital, Lisbon, from January 2007 to December 2009. São Francisco Xavier Hospital is a central and university hospital of Lisbon, which belongs to a 900 beds Hospital Centre serving a population of about 935,000 people as a tertiary referral center.

The MRSA isolates were collected mainly from bloodstream, from respiratory tract (tracheal aspirate and bronchoalveolar lavage) and from synovial fluid.

Only one isolate per patient was included in this analysis. For those patients with more than one isolate, only the first isolate was tested. All isolates were identified as Staphylococcus aureus according to standard methods.

\section{Antibiotics evaluated}

MICs of vancomycin and oxacillin were determined by the Etest. Oxacillin testing was performed to confirm oxacillin resistance.

\section{MIC testing methods}

For each isolate prior to MIC testing, a single bead was aseptically removed from the Microbank vial and spread onto the surface of trypticase soy agar plates supplemented with $5 \%$ sheep blood. These plates were then incubated overnight $(18-24 \mathrm{~h})$ at $35^{\circ} \mathrm{C}$ in ambient air. Each isolate was subcultured and incubated overnight for a second time under the same conditions. From these plates, portions of three to five individual colonies were inoculated into $5 \mathrm{~mL}$ of physiologic serum and incubated for $18 \mathrm{~h}$. A 0.5 McFarland turbidity standard was used to streak the inoculums onto the surface of a $150 \mathrm{~mm}$ Mueller - Hinton II agar plate. The surface of the plate was allowed to dry for $15 \mathrm{~min}$ prior to Etest strip application.

The MIC testing was performed using the Etest method, following manufacturer's guidelines. The antibiotic Etest strips were applied to the agar surface using an Etest applicator and were not moved following application. The MICs were read in 24-48 h. The MIC testing of the organisms was performed over a period of 4 weeks in a single laboratory.

The susceptibility breakpoint was $2 \mathrm{mg} / \mathrm{L}$ for vancomycin. The breakpoint for oxacillin resistance was $4 \mathrm{mg} / \mathrm{L}$. Actual Etest MIC values were used for all calculations and analyses and not rounded up to the next highest traditional 2-fold MIC value.

\section{Statistical analysis}

Standard descriptive statistics were used. Continuous variables were reported as mean \pm standard deviation.

Continuous variables were analyzed using the parametric unpaired Student's $t$ test, the nonparametric MannWhitney $U$ test or Kruskal-Wallis $\mathrm{H}$ test, according to data distribution. Categorical variables were compared using the Chi-square test.

MIC trends over the 3 years were assessed using nonparametric methods. For the analysis of MIC trends over time, Chi-square test was used.

Tests were performed two-tailed and considered significant when $\mathrm{p}<0.05$. All statistical tests were performed using SPSS for Windows (version 16.0: SPSS, Chicago, IL, USA) [4].

\section{Results}

During the study period a total of 93 MRSA isolates from 93 patients were collected for analysis. Twentynine isolates $(31,2 \%)$ were recovered in 2007, 30 (32,3\%) in 2008 and 34 (36,6\%) during 2009. 
Table 1 Clinical and demographic characteristics from the methicilin-resistant Staphylococcus aureus $(\mathrm{N}=93)$

\begin{tabular}{lc}
\hline & $\mathbf{N = 9 3}$ \\
\hline Age, mean ( \pm SD) & $76,7 \pm 13,0$ \\
\hline Gender, F (\%) & $52(55,9 \%)$ \\
\hline Department, N (\%) & $44(47,3 \%)$ \\
\hline Emergency department & $27(29,0 \%)$ \\
\hline Medical ward & $18(19,3 \%)$ \\
\hline Intensive care unit & $4(4,3 \%)$ \\
\hline Surgical ward & \\
\hline MRSA, N (\%) & $53(57 \%)$ \\
\hline Blood cultures & $39(41,9 \%)$ \\
\hline Respiratory tract & $1(1,1 \%)$ \\
\hline Others
\end{tabular}

The majority of MRSA isolates (57\%) were collected from bloodstream and from respiratory tract $(41,9 \%)$ mainly from tracheal aspirate. Only one MRSA was collected from synovial fluid. Clinical and demographic characteristics are presented in Table 1.

The lowest MIC was $0.75 \mathrm{mg} / \mathrm{L}$ that was observed in only one isolate.

Nineteen isolates (20.4\%) had MIC $1 \mathrm{mg} / \mathrm{L}, 38$ (40,9\%) isolates had MIC $1.5 \mathrm{mg} / \mathrm{L}$ and 35 (37.6\%) had MIC $2 \mathrm{mg} / \mathrm{L}$. No MRSA isolate presented a MIC $>2 \mathrm{mg} / \mathrm{L}$.

The MIC distribution for vancomycin is displayed in Figure 1. During the 3 year study period, we observed a significant fluctuation in the rate of MRSA with a vancomycin MIC >1 mg/L (2007: 86.2\%, 2008: 93.3\%, 2009: 58.8\%). Overall MICs for vancomycin declined during the study period. The MIC trends appeared to either plateau or slightly increase between 2007 and 2008, $(\mathrm{p}=\mathrm{n} . \mathrm{s})$ but decreased in $2009(\mathrm{p}=0.002)$.

\section{Discussion}

Methicillin-resistant Staphylococcus aureus is a major pathogen of nosocomial infections and is associated is associated with high mortality rates and healthcare costs $[10,11]$.

Studies reporting vancomycin MIC creep with MRSA have produced conflicting results. Minimum inhibitory concentration creep over time has been noted in some studies but large multicentre surveillance studies have not reported the same type of findings over time [12-14].

The factors involved in the development of reduced susceptibility to vancomycin and subsequent "glycopeptide MIC creep" are not entirely elucidated, but recognition of the phenomenon is important since it may be a precursor to hVISA and VISA [15]. This phenomenon, MIC creep, is of clinical concern because poorer treatment outcomes have been associated with higher vancomycin MICs [9,16-20].

Some authors advocate that combining data from multiple centers can obscure trends that may exist in one institution [14]. Some of these studies utilize less-sensitive traditional susceptibility markers (e.g. percentages, MIC50, MIC90) in their analyses. Vancomycin MIC result also varies by method of testing with Etest results tending to be $0.5-1.5 \log _{2}$ dilutions higher than reference broth method [21-23]. On the other hand, combining data from multiple centers can obscure trends that may exist within a given institution or country as a result of differences in patient populations and drug usage patterns.

In our institution we were unable to find data compatible with the presence of vancomycin MIC creep among MRSA over a 3-year period, on the contrary we found a significant statistical decline in vancomycin MIC creep in 2009.

Our study had some limitations. First, this is a retrospective single center study, possibly introducing a selection bias. Second, the number of patients enrolled was small, making statistical calculations problematic. Third, MICs were only analyzed over a 3-year period, longer periods are needed to a broad statement about MIC

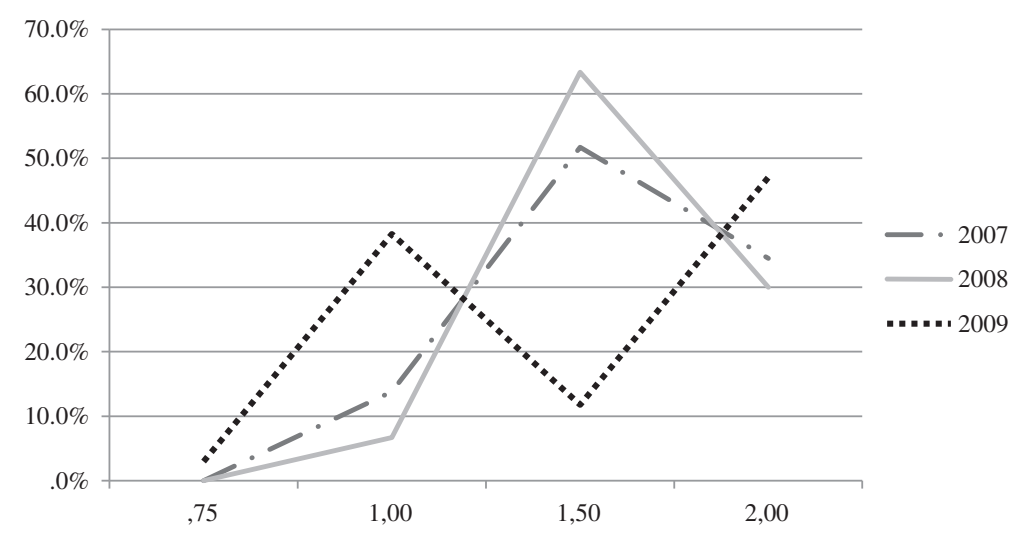

Figure 1 Vancomycin MIC population distribution 2007 - 09. 
trends. However this is the first Portuguese study and in this study we analyzed only one isolate per patient and only isolates in significative samples were included (colonizations were not analyzed).

\section{Conclusions}

In conclusion, though the cause of vancomycin "MIC creep" is unknown, decreased MRSA susceptibility to vancomycin is likely due to overuse as well as to suboptimal vancomycin dosing. The phenomenon known as MIC creep has been observed only in the last 20 years. Critical evaluation of site of infection, MIC data, aggressive dosing with close monitoring and follow up is warranted with vancomycin therapy. We also advise that all hospitals should monitor their local status of vancomycin MICs in invasive MRSA isolates to screen for the possibility of MIC creep.

\section{Competing interests}

J.S., E.G. and F.M. have no conflicts of interest to declare. P.P. has received honoraria and served as advisor of Gilead, Merck Sharp \& Dohme and Pfizer.

\section{Authors' contributions}

JS designed the study, collected the data and wrote the manuscript, EG collected the data and reviewed the manuscript, FM reviewed the manuscript, PP designed the study and reviewed the manuscript. All authors read and approved the final manuscript.

\section{Author details}

${ }^{1}$ Polyvalent Intensive Care Unit, São Francisco Xavier Hospital, CHLO, Lisbon, Portugal. ${ }^{2}$ CEDOC, Faculty of Medical Sciences, New University of Lisbon, Lisbon, Portugal. ${ }^{3}$ Microbiology Laboratory, Clinical Pathology Department, São Francisco Xavier Hospital, CHLO, Lisbon, Portugal.

Received: 22 November 2012 Accepted: 7 February 2013 Published: 19 February 2013

\section{References}

1. Styers D, Sheehan DJ, Hogan P, Sahm DF: Laboratory-based surveillance of current antimicrobial resistance patterns and trends among Staphylococcus aureus: 2005 status in the United States. Ann Clin Microbiol Antimicrob 2006, 5:2.

2. Johnson AP: Methicillin-resistant Staphylococcus aureus: the European landscape. J Antimicrob Chemother, 66(Suppl 4):iv43-iv48.

3. European Centre for Disease Prevention and Control: Antimicrobial resistance surveillance in Europe 2010. Annual Report of the European Antimicrobial Resistance Surveillance Network (EARS-Net). Stockholm: ECDC; 2011.

4. Melo-Cristino JAAF, Amorim JM, Diogo J, Lito LM, Lopes P, Marques J, Marques T, Martins F, Monteiro L, Nascimento I, Pessanha MA, Piedade J, Ramos MH, Ribeiro G, Rodrigues A, Salgado MJ, Sobral L, Spencer D: Estudo multicê ntrico de resistê ncia aos antimicrobianos em nove hospitais portugueses. Comparaç ã o de resultados num intervalo de uma dé cada. Revista Portuquesa de Doenç as Infecciosas 2006, 3:7-15.

5. Jones RN: Microbiological features of vancomycin in the 21 st century: minimum inhibitory concentration creep, bactericidal/static activity, and applied breakpoints to predict clinical outcomes or detect resistant strains. Clin Infect Dis 2006, 42(Suppl 1):S13-S24.

6. Liu C, Bayer A, Cosgrove SE, et al: Clinical practice guidelines by the infectious diseases society of america for the treatment of methicillinresistant Staphylococcus aureus infections in adults and children: executive summary. Clin Infect Dis 2011, 52:285-92.

7. Maclayton DO, Suda KJ, Coval KA, York CB, Garey KW: Case-control study of the relationship between MRSA bacteremia with a vancomycin MIC of $2 \mathrm{microg} / \mathrm{mL}$ and risk factors, costs, and outcomes in inpatients undergoing hemodialysis. Clin Ther 2006, 28:1208-1216.
8. Moise-Broder PA, Sakoulas G, Eliopoulos GM, Schentag JJ, Forrest A, Moellering RC Jr: Accessory gene regulator group II polymorphism in methicillin-resistant Staphylococcus aureus is predictive of failure of vancomycin therapy. Clin Infect Dis 2004, 38:1700-1705.

9. Sakoulas G, Moise-Broder PA, Schentag J, Forrest A, Moellering RC Jr, Eliopoulos GM: Relationship of MIC and bactericidal activity to efficacy of vancomycin for treatment of methicillin-resistant Staphylococcus aureus bacteremia. J Clin Microbiol 2004, 42:2398-2402.

10. Shorr AF, Combes A, Kollef MH, Chastre J: Methicillin-resistant Staphylococcus aureus prolongs intensive care unit stay in ventilatorassociated pneumonia, despite initially appropriate antibiotic therapy. Crit Care Med 2006, 34:700-706.

11. Tverdek FP, Crank CW, Segreti J: Antibiotic therapy of methicillin-resistant Staphylococcus aureus in critical care. Crit Care Clin 2008, 24:249-260. vii-viii.

12. Alos JI, Garcia-Canas A, Garcia-Hierro P, Rodriguez-Salvanes F: Vancomycin MICs did not creep in Staphylococcus aureus isolates from 2002 to 2006 in a setting with low vancomycin usage. J Antimicrob Chemother 2008, 62:773-775.

13. Ho PL, Lo PY, Chow KH, Lau EH, Lai EL, Cheng VC, Kao RY: Vancomycin MIC creep in MRSA isolates from 1997 to 2008 in a healthcare region in Hong Kong. J Infect 2010, 60(2):140-145.

14. Steinkraus G, White R, Vancomycin FL: Vancomycin MIC creep in nonvancomycin-intermediate Staphylococcus aureus (VISA), vancomycinsusceptible clinical methicillin-resistant $\mathrm{S}$. aureus (MRSA) blood isolates from 2001-05. J Antimicrob Chemother 2007, 60:788-794.

15. Appelbaum PC: Reduced glycopeptide susceptibility in methicillinresistant Staphylococcus aureus (MRSA). Int J Antimicrob Agents 2007, 30:398-408.

16. Hidayat LK, Hsu DI, Quist R, Shriner KA, Wong-Beringer A: High-dose vancomycin therapy for methicillin-resistant Staphylococcus aureus infections: efficacy and toxicity. Arch Intern Med 2006, 166:2138-2144

17. Lodise TP, Graves J, Evans A, et al: Relationship between vancomycin MIC and failure among patients with methicillin-resistant Staphylococcus aureus bacteremia treated with vancomycin. Antimicrob Agents Chemother 2008, 52:3315-3320.

18. Moise PA, Sakoulas G, Forrest A, Schentag JJ: Vancomycin in vitro bactericidal activity and its relationship to efficacy in clearance of methicillin-resistant Staphylococcus aureus bacteremia. Antimicrob Agents Chemother 2007, 51:2582-2586.

19. Soriano A, Marco F, Martinez JA, et al: Influence of vancomycin minimum inhibitory concentration on the treatment of methicillin-resistant Staphylococcus aureus bacteremia. Clin Infect Dis 2008, 46:193-200.

20. Choi EY, Huh JW, Lim CM, Koh Y, Kim SH, Choi SH, Kim YS, Kim MN, Hong SB: Relationship between the MIC of vancomycin and clinical outcome in patients with MRSA nosocomial pneumonia. Intensive Care Med 2010, 37(4):639-647.

21. Sader HS, Rhomberg PR, Jones RN: Nine-hospital study comparing broth microdilution and Etest method results for vancomycin and daptomycin against methicillin-resistant Staphylococcus aureus. Antimicrob Agents Chemother 2009, 53:3162-3165.

22. Prakash V, Lewis JS 2nd, Jorgensen JH: Vancomycin MICs for methicillinresistant Staphylococcus aureus isolates differ based upon the susceptibility test method used. Antimicrob Agents Chemother 2008, 52:4528.

23. Leonard SN, Rossi KL, Newton KL, Rybak MJ: Evaluation of the Etest GRD for the detection of Staphylococcus aureus with reduced susceptibility to glycopeptides. J Antimicrob Chemother 2009, 63:489-492.

doi:10.1186/1756-0500-6-65

Cite this article as: Joana et al:: Is vancomycin MIC creep a worldwide phenomenon? Assessment of $S$. aureus vancomycin MIC in a tertiary university hospital. BMC Research Notes 2013 6:65. 